Revista General de Información y Documentación ISSN: $1132-1873$

\title{
Resultados de la gestión archivística en el Sistema de Archivos de la congregación religiosa católica de los Padres Sacramentinos en España.
}

\author{
Édison Yamir Toledo Díaz ${ }^{1}$
}

Recibido: 18 de abril 2016 / Aceptado: 8 de octubre de 2016

Resumen. El artículo aborda los principales resultados obtenidos en la realización de la gestión archivística mediante las fases de identificación, valoración, organización y descripción de los fondos documentales que tiene el Sistema de Archivos de los Padres Sacramentinos en España, compuesto por un Archivo Provincial y cuatro Archivos Locales. En el trabajo se demuestra de forma práctica y vivencial, que es perfectamente posible la aplicación de los fundamentos teóricos, metodológicos y prácticos de la Archivística en este tipo de Archivos.

Palabras claves: Identificación; Valoración, Organización; Clasificación; Ordenación; Instalación; descripción; Gestión archivística; Archivos privados religiosos católicos; Congregación religiosa; Padres Sacramentinos.

\section{[en] Outcome of archival management in the Archives System of the Catholic religious congregation of Priests of the Blessed Sacrament in Spain.}

\begin{abstract}
The article analyzes the main results obtained in the archival processing through the phases of identification, appraisal, organization and description of the documentary holdings of Congregation of the Blessed Sacrament in Spain, which consists of one Provincial Archives and four local archives. This study summarized that it is perfectly possible to apply the theoretical, methodological and practical basis of the Archival science in this kind of archival institutions.

Keywords: Identification; Appraisal; Organization; Archival classification; Arrangement; Storage; Archival description; Archival processing; Private catholic archives; Religious congregations; Blessed Sacrament priest.
\end{abstract}

Sumario. 1. Introducción. 2. Apuntes sobre la historia de la institución de los padres sacramentinos de la congregación del santísimo sacramento en España y sus trabajos archivísticos previos. 3. El enfoque de sistema en los archivos de los padres sacramentinos en España. 4. Conclusiones. 5. Referencias bibliográficas.

Cómo citar: Toledo Díaz, E. Y. (2016) Resultados de la gestión archivística en el Sistema de Archivos de la congregación religiosa católica de los Padres Sacramentinos en España, en Revista General de Información y Documentación 26 (2), 455-480.

1 Archives Nationales de Luxembourg.

Email: edison.yamir@gmail.com 


\section{Introducción}

En julio del año 2011, los Padres Sacramentinos de la Provincia de San Pedro Apóstol iniciaron un proceso de reorganización de su Sistema de Archivos compuestos por cuatro Archivos Locales y uno Provincial, distribuidos entre las ciudades de Madrid, Tolosa y Pamplona (España).

Para enfrentar esta actividad, hubo que recopilar información e indagar sobre trabajos previos en Archivos de congregaciones religiosas. Entre otros trabajos, se consultó el publicado por Farías (2005) con el título, "Archivos Eclesiásticos y Religiosos: Límites y desafíos ante la Sociedad de la Información y el Conocimiento", que posibilitó un acercamiento a algunas tendencias actuales de la organización archivística en archivos conventuales ante los retos de la sociedad del siglo XXI.

Durante ese periodo, también se consultó el excelente trabajo del profesor Mariano García Ruipérez, titulado "Tipologías. Series documentales. Cuadros de clasificación. Cuestiones metodológicas y prácticas". En esta obra, el autor enunciaba que "...los archivos de órdenes religiosas no reflejan una estructura de clasificación con criterios archivísticos actuales". Esta idea, se convirtió en la motivación esencial y a la vez en la problemática a resolver durante todo el trabajo de reorganización archivística de los Archivos de los Padres Sacramentinos en España.

Pero..., ¿por qué reorganización archivística? La idea consistía en retomar lo realizado previamente de manera empírica por los propios Padres Sacramentinos, sirviendo de base para el nuevo trabajo a emprender, esta vez bajo un enfoque fundamentado en la disciplina archivística ya que la consulta bibliográfica evidenciaba que la organización archivística en archivos del clero regular había tenido mucho de improvisación e innovación particulares, a veces muy alejadas de la Archivística.

Es preciso plantear primero que los Padres Sacramentinos pertenecen a la Congregación del Santísimo Sacramento. Ésta, es un instituto religioso perteneciente al clero regular de la Iglesia Católica cuya misión principal es, según su Regla de Vida, "poner en el centro de su vida y de la vida de los fieles, la Eucaristía, celebrada en la verdad e interiorizada en una oración de adoración y de contemplación".

Pero... ¿pueden los Archivos de un instituto religioso organizarse o reorganizarse siguiendo los fundamentos teóricos y prácticos que plantea la Archivística? La respuesta es más que evidente. Si es Archivo, lo precisará aunque tendrá particularidades que contextualizarán esta práctica específica. Lo anterior no es una conclusión anticipada de este trabajo sino la concepción con la que se concibió la realización del mismo en este tipo de Archivos .

Por ello, el trabajo pretende en todo momento señalar la forma en que se realizó el proceso de reorganización de estos Archivos. Se advierte que habrá algunas especificidades que adquiere el mismo pero prima la idea de que la reorganización de este tipo de Archivos, no es una excepción dentro de la gestión, procesamiento o tratamiento archivísticos.

El hecho de que tenga especificidades es tan normal como lo es la propia necesidad de contextualizar los fundamentos de todo tipo de la Archivística al tipo de fondos y de Archivos en los que se apliquen. Este es por tanto un texto con 
marcado carácter vivencial y práctico-funcional. En él no predominan definiciones ni disertaciones sobre archivo, documento, documento de archivo, gestión documental, gestión archivística ni gestión del Archivo: no es la intención de estas líneas. Tampoco se harán nuevas menciones a qué es un fondo, una serie, una tipología documental o qué son unidades documentales simples o compuestas.

La finalidad de estas líneas no es la de incrementar el número de definiciones dadas para los conceptos anteriores, máxime cuando son abordados, -solo en el habla castellana y sin despreciar a los anglosajones y francófonos-, por teóricos e investigadores de la Archivística como lo son Antonia Heredia, José Ramón Cruz Mundet, Mariano García Ruipérez, Vicenta Cortés, Ramón Alberch, José Luis La Torre, Aurelio Tanodi, Ana Duplá y demás.

Lo que sí se encontrará en este trabajo es una aplicación práctica y contextualizada de las concepciones archivísticas de los autores anteriores, que desde una relativa y subjetiva heterogeneidad, es posible integrarlas al procesar fondos privados y religiosos. El trabajo es además un ejemplo de cómo llevar a la práctica de forma armónica la identificación, valoración, organización (ordenación y clasificación) y descripción mediante la Norma $\operatorname{ISAD}(\mathrm{G})$ de fondos y series documentales en Archivos privados y religiosos, respetando el principio de procedencia y el del orden natural de los documentos.

Aunque se explicará más adelante, los Archivos de los Padres Sacramentinos en España están regidos desde enero del año 2012 por la Delegación de España de la Provincia de Nuestra Señora del Santísimo Sacramento en Italia, pero sea como fuere, los Archivos son el resultado de una institución preocupada por la conservación y preservación de una producción documental muy valiosa. Esta testimonia el quehacer de los Padres Sacramentinos en España, Colombia, Puerto Rico y Cuba. Sus Archivos, son el resultado además de la preocupación y el celo de un grupo de religiosos que desde siempre reconocieron la importancia y trascendencia de conservar los documentos.

Estos sirven de testimonio y reflejo de una institución única, que tiene y realiza funciones inherentes a un instituto religioso, con gestiones y trámites propios del ejercicio de su actividad. Gracias a la perseverancia de esos religiosos, es que ha llegado a nuestros días un riquísimo patrimonio documental, quizás no tan grande en volumen como sí en historia, que refleja el devenir de esta institución y de sus personas.

\section{Apuntes sobre la historia de la institución de los padres sacramentinos de la congregación del santísimo sacramento en españa y sus trabajos archivísticos previos}

Los Padres Sacramentinos llegaron a la localidad de Tolosa en Gipuzkoa en 1907. Fundaron allí la primera de sus comunidades en tierras ibéricas. Junto a las fundadas luego en Madrid (1942) y Pamplona (1944) se erigió canónicamente la Vice-Provincia de España en 1946. Esta institución, tuvo una vida muy efímera, siendo sustituida en 1949 por la Provincia de San Pedro Apóstol, institución que a su vez dio paso en enero de 2012 a la actual Delegación. 
La Congregación del Santísimo Sacramento, popularmente conocida como "Padres Sacramentinos" e identificados con las siglas "SSS", fue fundada en París en el año 1856 por el Padre Pedro Julián Eymard. En la actualidad, la Congregación del Santísimo Sacramento está dirigida por un Padre Superior General que reside en Roma, quien es asistido por un Consejo General. De estos dependen todas las provincias, regiones o delegaciones religiosas sacramentinas. Estas son las formas de organización y dirección del personal empleadas por la Congregación.

Cada Provincia, Región o Delegación es dirigida por un Superior Provincial, Regional o Delegado, respectivamente, con sus también respectivos Consejos, salvo los Delegados. Las Provincias, Regiones o Delegaciones no se identifican con una denominación geográfica sino con el nombre de un santo protector u otro nombre religioso. Es por ello que por ejemplo, una misma provincia sacramentina generalmente está presente en varias regiones geográficas a la vez. Tal fue el caso de la Provincia de San Pedro Apóstol de la Congregación del Santísimo Sacramento, cuyas áreas de incidencia fueron la Península Ibérica (España), parte de América Latina y el Caribe.

Como ya se enunció, en el año 1946 lo que eran tres comunidades sacramentinas en Tolosa, Madrid y Pamplona, se convirtieron en la Vice-Provincia de España por decreto del Consejo General de la Congregación del Santísimo Sacramento y firmado por el Superior General. Tres años más tarde, por decreto $\mathrm{N}^{\mathrm{o}}$ 2831/49 de fecha 8 de mayo de 1949 firmado también por el Superior General de la Congregación, se constituían canónicamente las comunidades de la Congregación que existían ya en España en una Provincia con el nombre de San Pedro Apóstol. A las tres primeras casas ya existentes en la Provincia, se les unieron las siguientes fundaciones:

- Comunidad de Villaro SSS (Bizkaia, España) el 27 de septiembre de 1950.

- Comunidad de La Habana SSS (Cuba) el 8 de abril de 1953.

- Comunidad de Bogotá SSS (Colombia) el 25 de diciembre de 1956.

- Comunidad de Popayán SSS (Cauca, Colombia) el 27 de febrero de 1957.

- Comunidad de Bilbao-Zurbaran SSS (Bizkaia, España) el 7 de octubre de 1958.

- Comunidad de Medellín SSS (Antioquia, Colombia) el 10 de diciembre de 1960.

- Comunidad de Madrid-Santa Hortensia SSS (Madrid, España) el 26 de marzo de 1966.

- Comunidad de Barcelona SSS (España) el 25 de mayo de 1967.

- Comunidad de Caguas SSS (Puerto Rico) el 5 de agosto de 1967.

- Comunidad de Bilbao-Deusto SSS (Bizkaia, España) el 29 de septiembre de 1968.

- Comunidad de Madrid-Corazón de María SSS (Madrid, España) el 6 de octubre de 1969.

- Comunidad de Cali SSS (Valle del Cauca, Colombia) el 24 de abril de 1970.

- Comunidad de Écija SSS (Sevilla, España) el 27 de marzo de 1995.

La estructura de dirección tanto de la Institución más general como de las propias Comunidades seguía el esquema tradicional, vertical y descendente. No obstante, el Consejo Provincial y el Capítulo Provincial, eran órganos colegiados de dirección y 
de toma de decisiones compartidas. La Provincia desarrolló desde el año 1949 en que se erigió como tal y hasta el año 2011, 17 Capítulos Provinciales.

Desde finales del año 2011, los planes de la Provincia eran los de integrarse a la Provincia de Nuestra Señora del Santísimo Sacramento en Italia. A partir de enero de 2012, dejaría de ser una Provincia y se constituiría en la Delegación de España. Esa sería la nueva institución que heredaría el Sistema de Archivos de los Padres Sacramentinos en España. En dicho Sistema, múltiples son las evidencias que demuestran el interés del Vice-Provincial primero, de los Provinciales de San Pedro Apóstol luego y de los actuales Delegados, por guardar y custodiar los documentos que se creaban o se recibían durante el ejercicio de sus respectivos cargos al frente de los religiosos.

En el inicio del tratamiento de los fondos en las distintas Comunidades se pudo comprobar que los documentos se archivaban con dos de las tradicionales finalidades de cualquier Archivo. Por un lado, la de contar con los documentos como pruebas documentales que testificaran $\mathrm{y}$ aún testifican la gestión desempeñada. Por otro lado, para el propio ejercicio de gobierno por los Superiores en la dirección de las personas. Éstas últimas, a su vez, en reiteradas ocasiones necesitaban documentos que para su elaboración requerían nuevamente de la consulta de los documentos.

Con estos antecedentes, el hecho real es que resulta manifiesto el interés y la necesidad de los Padres Sacramentinos de contar con sus Archivos y conservarlos. Constan evidencias de la organización, cuidado, uso y control de estos desde la época en la que sólo existía la Comunidad de Tolosa SSS. También se ha podido comprobar, que la conservación de los documentos era asumida por los propios Superiores. Estos asumían la responsabilidad como una tarea más inherente a su cargo. Algunos ejemplos que ilustran el interés por archivar los documentos son:

- La frase "Archivo Provincial" escrita en el borde superior de múltiples documentos, lo que evidencia el interés por el destino que tendrían estos. (Ver Figura 1).
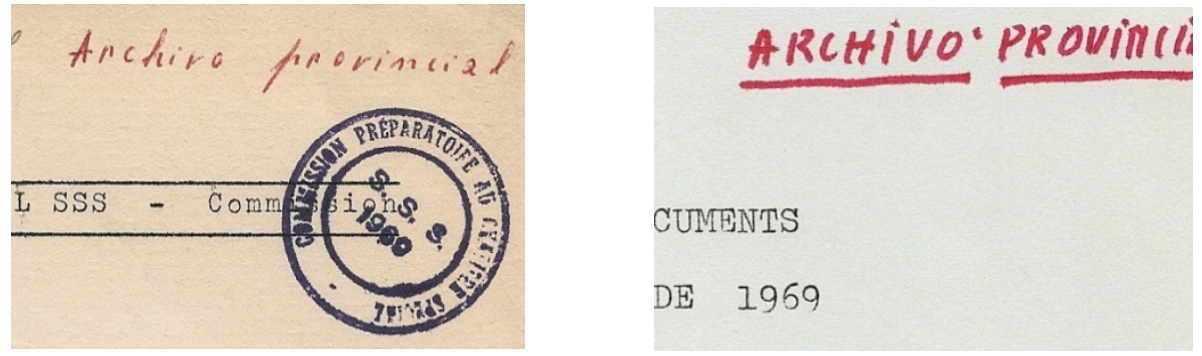

Figura 1. Fragmentos de documentos que ejemplifican el interés por dejar constancia de ellos en el Archivo Provincial.

Fuente: Archivo Provincial de los Padres Sacramentinos. Julio de 2011. 
- Documentos en cuya confección se hace mención al uso del Archivo. (Ver Figura 2.)

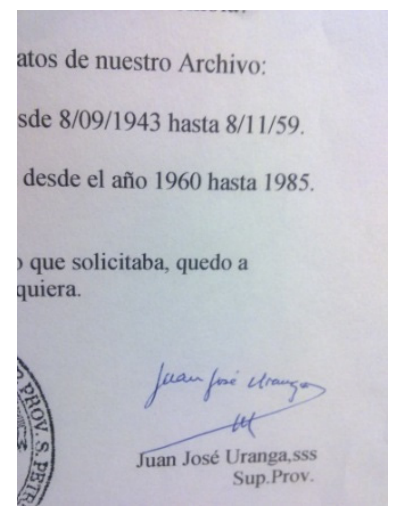

Figura 2. Fragmento de un documento firmado por un Provincial, que hace mención en su confección al uso del Archivo.

Fuente: Archivo Provincial de los Padres Sacramentinos. Abril de 2012.

- Trabajos previos desarrollados con series documentales nombradas "Expedientes Personales" y "Capítulos", con esquemas de numeración continua. (Ver Figura 3).
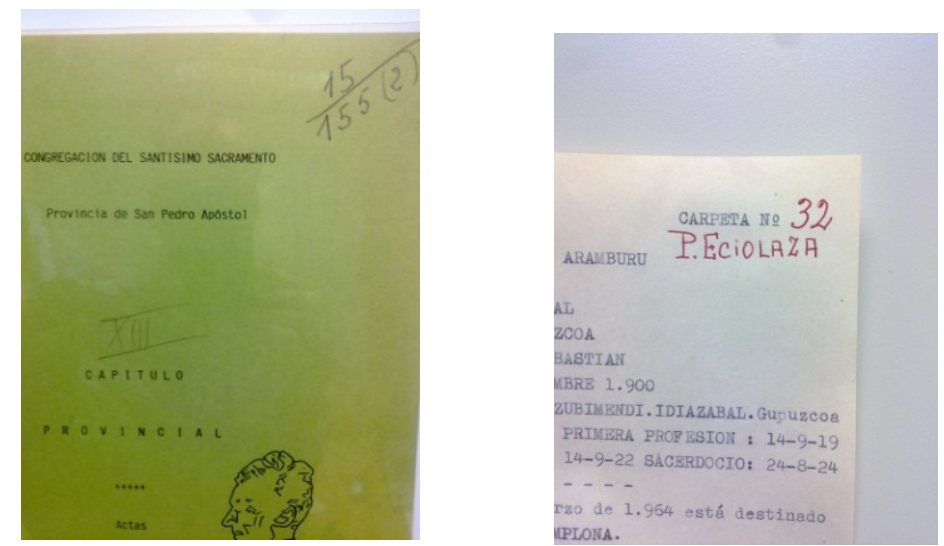

Figura 3. Ejemplos de numeraciones continuas usadas para documentos ordenados dentro de series documentales.

Fuente: Archivo Provincial de los Padres Sacramentinos. Abril de 2012.

El ejemplo anterior, sin estar exento de deficiencias, ha sido uno de los mejores encontrados desde el punto de vista archivístico. Consistió en formas de identificar grupos documentales para luego localizarlos en las estanterías. Constan evidencias de que fue un método que funcionó aunque solo fuera para expedientes personales. No obstante, a veces se hicieron coincidir más de un método de numeración y no se 
han encontrado en la actualidad los instrumentos que permitieran ejecutar con esos sistemas numéricos anteriores.

- Aun primando la agrupación de documentos por materias y no por la función, trámite o gestión, se aprecia un intento serio de ordenación documental.

Las evidencias del ejemplo anterior son los conjuntos de documentos identificados como "Capítulos Provinciales" o "Consejos Provinciales", que han contribuido en la actualidad a la identificación de algunos de los sujetos productores y de las series documentales dentro de los fondos. (Ver Figura 4).

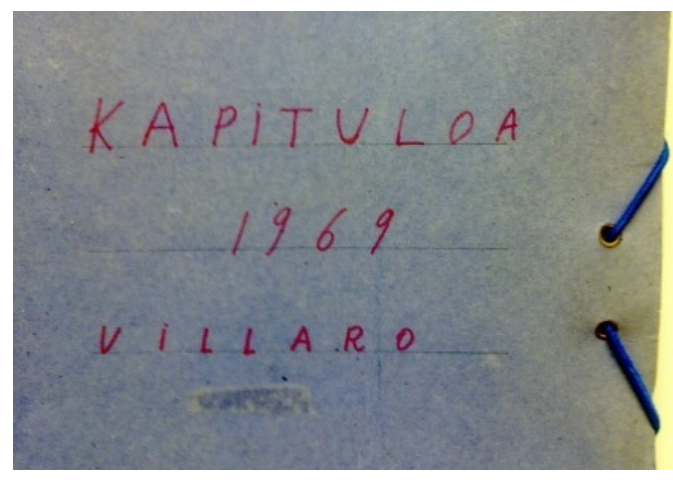

Figura 4. Fragmento de una carpeta denominada "Kapituloa" que agrupaba un conjunto de documentos por su materia.

Fuente: Archivo Provincial de los Padres Sacramentinos. Abril, 2012.

Lo anterior, a pesar de constituir un noble intento, está desaconsejado por la práctica archivística. No es correcto agrupar los documentos por temática o materia sino que es necesario hacerlo por gestiones, trámites o funciones inherentes a la institución productora. De hecho es ésta una de las diferencias de la Archivística con otras ciencias documentales. No obstante, es ineludible reconocer que el haber agrupado los documentos por temática, si bien no es ortodoxo desde el punto de vista archivístico, al menos evitó la dispersión, el desorden y la pérdida de los documentos. Por lo que en parte facilitó el proceso posterior de reorganización. 
- La existencia de instrumentos de búsqueda de información denominados "catálogos", por ejemplo, de expedientes personales. (Ver Figura 5).
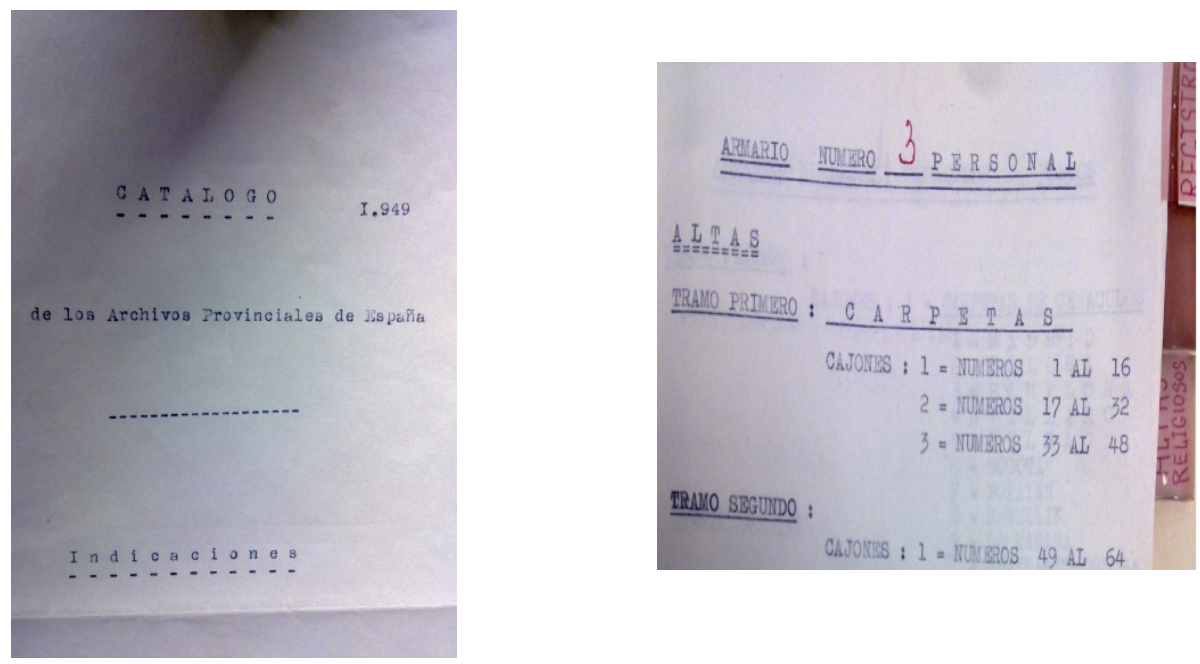

Figura 5. Ejemplos de instrumentos denominados "Catálogos", empleados en el Archivo Provincial.

Fuente: Archivo Provincial de los Padres Sacramentinos. Abril, 2012.

Los anteriores constituyen antecedentes valiosos a pesar de su empirismo, siendo justo reconocer el esfuerzo. En algunos casos no se encontró luego la correspondencia entre los instrumentos diseñados antaño y la instalación de los documentos. Algunas de las causas que parecen haber incidido en ello fueron las obras constructivas, los movimientos internos, los cambios de locales, el deterioro de las carpetas, entre otras.

- Orientaciones recibidas de instancias superiores para la organización de los Archivos. (Ver Figura 6).

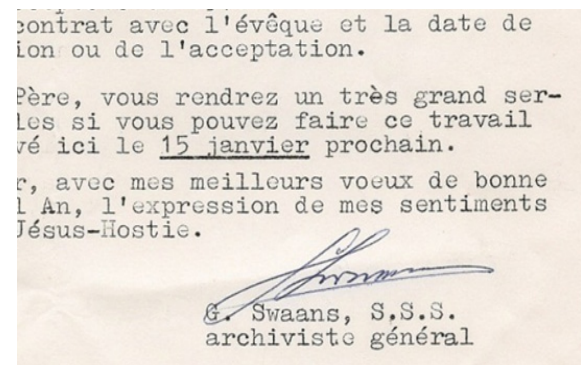

Figura 6. Fragmento de un documento enviado por el Archivero General de la Congregación del Santísimo Sacramento a la Provincia de San Pedro Apóstol con orientaciones precisas para el Archivo.

Fuente: Archivo Provincial de los Padres Sacramentinos. Julio de 2011. 
- La seriedad en el cuidado de los Archivos y sus traspasos mediante actas firmadas de un responsable saliente a otro (Ver Figura 7).

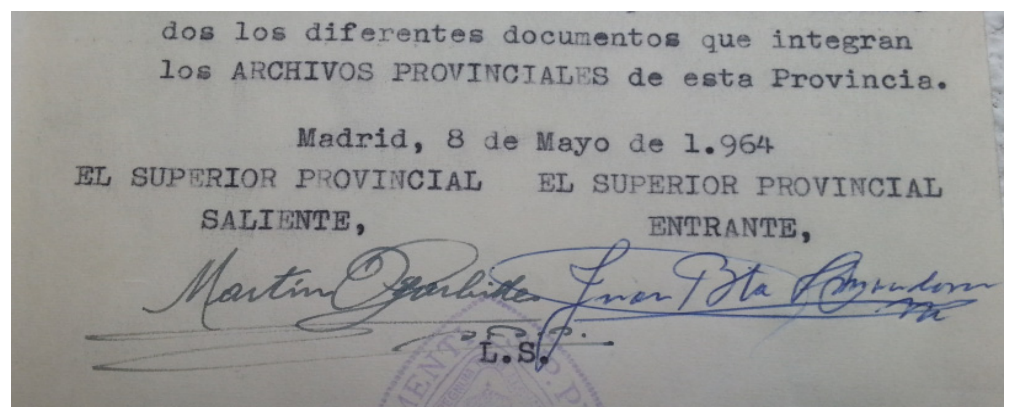

Figura 7. Fragmento de un acta de entrega del Archivo Provincial entre Provinciales de San Pedro Apóstol.

Fuente: Archivo Provincial de los Padres Sacramentinos. Julio de 2015.

Cualesquiera que hayan sido las razones que primaron en la conservación del patrimonio documental realizado por los Superiores, el hecho real es que permitió que los documentos hayan llegado a los días de hoy en perfecto estado de conservación. Eso, desde el punto de vista archivístico es ya digno de resaltar y agradecer por la importancia que tiene para la preservación de la memoria histórica de la institución.

Con ello se ayuda a preservar la historia de una institución que se inició como Vice-Provincia de España, continuó como Provincia de San Pedro Apóstol y hoy es la Delegación de España de la Provincia de Nuestra Señora del Santísimo Sacramento. Ello ayudará también a conservar los documentos que han generado, recibido y custodiado los Padres Sacramentinos en su devenir histórico desde 1946. Ellos constituyen los garantes históricos de la presencia sacramentina en la península ibérica pero también en Colombia, Puerto Rico y Cuba, de ahí la necesidad de preservarlo con un proceso de reordenación archivística.

\section{El enfoque de sistema en los archivos de los padres sacramentinos en España}

La reorganización efectuada mediante el tratamiento, procesamiento o gestión archivística del Sistema de Archivos de los Padres Sacramentinos se desarrolló entre los años 2011 y 2016. El trabajo partió reconociendo la concepción de que este tratamiento es un proceso de tipo sistémico. A su vez se entiende como un grupo secuencial de acciones, tareas y/o procedimientos interrelacionados que conducen a un fin u objetivo. Pero también hay que entenderlo como la manera de 
obtener un resultado con una nueva cualidad, que sólo se alcanzará si cada elemento componente del sistema ofrece sus cualidades intermedias .

De tal manera, las fases de identificación, valoración, organización y descripción de los documentos, en tanto subprocesos sistémicos, por sí solos no constituyen un proceso archivístico acabado y completo, sino partes del mismo. Al realizarlos de conjunto, conducen a la conservación permanente del documento de archivo, a su acceso, utilización y consulta por los usuarios.

El fin último es el acceso a los documentos. Como tal esa será su nueva cualidad en el tipo de Archivos que nos ocupa. Esta nueva cualidad no puede lograrse solo con el hecho de identificar los productores, organizar los documentos, describir las series o valorar los documentos. De lo que se trata es de la realización consciente de cada uno de los procedimientos o pasos anteriores de forma procesal y sistémica. Ello no quiere decir hacerlo de una forma seccionada ni fragmentada sino interrelacionadas e interindependientes. Esto es realizable al menos en Archivos privados y religiosos en los que se realice la reorganización de todo el volumen documental.

Lo anterior determinó que las denominadas fases del tratamiento, proceso, procesamiento o gestión archivística, no se realizaran de forma exclusiva en la práctica. En más de una ocasión hubo que recurrir a la identificación cuando se describía o a la valoración cuando se clasificaba, entre otras interrelaciones, lo que no quiere decir tampoco que cada parte del proceso no tenga su propia identidad.

Con estas premisas anteriores, la identificación, valoración, organización, descripción, y la conservación de los documentos en los Archivos, se realizaron en secuencias ordenadas pero alternantes y concebidas desde un inicio de forma interrelacionadas, aunque para mostrar y explicar los resultados obtenidos en cada fase o momento, hubiera que hacerlo luego de forma independiente como se verá a continuación.

La identificación fue la fase que se implementó en un primer momento dentro del procesamiento archivístico de los fondos documentales de los Padres Sacramentinos. En esta fase se investigaron en cada una de las instancias de toda la institución (Comunidades, Provincia, Delegación), sus funciones y categorías organizativas que en mayor grado, determinan las archivísticas. Fue un proceso intelectual que partió del estudio de las normas internas que aseguraron y aseguran a la Institución su estatus y misión.

Como conclusión de esta fase se identificaron las funciones y categorías de cada comunidad sacramentina en particular, de la Provincia y luego de la Delegación, diagnosticándose también el flujo documental y de información. En cada instancia se reiteró que ese flujo tiene una orientación vertical y bidireccional. Un ejemplo global de ese flujo se puede apreciar en el organigrama general de la institución con todas sus instancias subordinadas y entidades relacionadas que se aprecia en la Figura 8. 


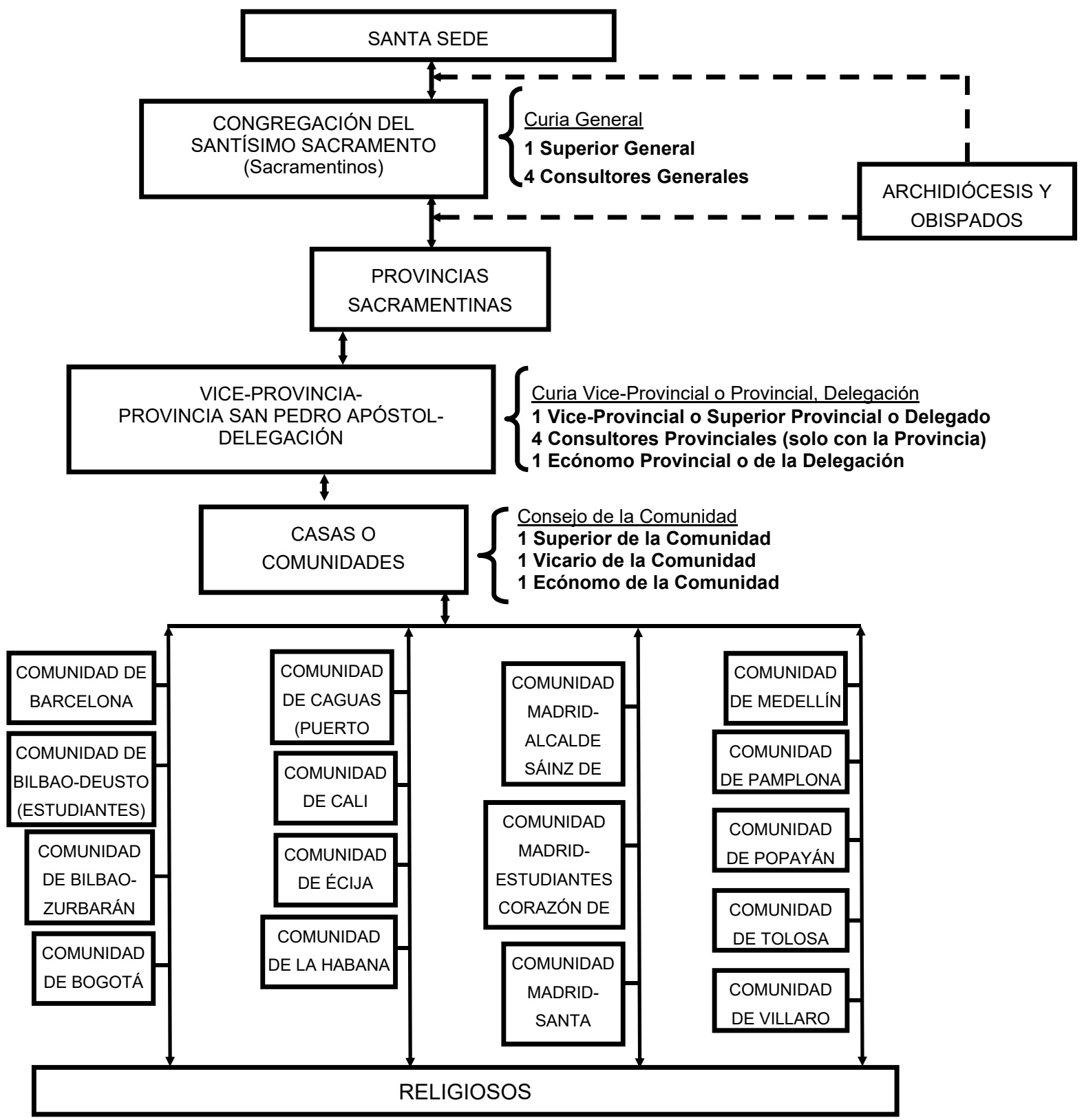

Figura 8. Organigrama de la institución que ha dirigido a los religiosos sacramentinos en España, Colombia, Puerto Rico y Cuba y que refleja la jerarquía organizacional y el flujo documental resultante.

Fuente: Toledo Díaz, Edison. Elaboración propia, mayo de 2015. 
En cada Comunidad se elaboró un organigrama similar al de la Figura 8 pero ajustado a su nivel particular. Ello, junto al análisis y revisión de los documentos, de los productores de los mismos, de las tipologías documentales y de indagaciones empíricas, propiciaron la determinación de la estructura general de cada Archivo en cuestión.

Además, el análisis de los elementos anteriores propios de la identificación, condujeron a la obtención de las categorías archivísticas que son un reflejo de la estructura interna de los fondos y posteriormente lo sería de sus series.

En la Tabla 1 se ilustra un ejemplo de la estructura de los fondos, en este caso en particular, los del Archivo Provincial de los Padres Sacramentinos en Madrid.

Tabla 1. Estructura de los fondos del Archivo Provincial de los Padres Sacramentinos determinada durante el proceso de identificación.

\section{ARCHIVO PROVINCIAL DE LOS PADRES SACRAMENTINOS, MADRID}

\section{Fondo Vice-Provincia}

2. Fondo Provincia de San Pedro Apóstol

- Sección: Función de gobierno

- Sección: Función de dirección de personal

- Sección: Función de realización de actividades

- Sección: Función de gestión económica

- Sección: Función de gestión del patrimonio

- Sección: Función de pago de impuestos y tributaciones

- Sección: Función de control de ingresos y gastos

- Subfondo: Comunidad de Barcelona SSS

- Subfondo: Comunidad de Bilbao-Deusto SSS

- Subfondo: Comunidad de Bilbao-Zurbaran SSS

- Subfondo: Comunidad de Bogotá SSS

- Subfondo: Comunidad de Caguas SSS

- Subfondo: Comunidad de Cali SSS

- Subfondo: Comunidad de Écija SSS

- Subfondo: Comunidad de La Habana SSS

- Subfondo: Comunidad de Madrid-Sáinz de Baranda SSS

- Subfondo: Comunidad de Madrid-Santa Hortensia SSS

- Subfondo: Comunidad de Madrid-Corazón de María SSS

- Subfondo: Comunidad de Medellín SSS

- Subfondo: Comunidad de Pamplona SSS

- Subfondo: Comunidad de Popayán SSS

- Subfondo: Comunidad de Tolosa SSS

- Subfondo: Comunidad de Villaro SSS

- Subfondo: Región Juan XXIII

3.- Fondo: Delegación de la Provincia de Nuestra Señora del Santísimo Sacramento

- Función de gobierno

- Función de gestión económica

- Función de participación en actividades

Fuente: Toledo, Edison, mayo 2015. 
Se partía de la concepción de que en un fondo se custodian los documentos generados y recibidos por una institución o persona (productor) en el ejercicio de sus funciones y a lo largo del tiempo. Ese productor puede estar formado a su vez por uno o varios sujetos productores. Lo que se ha perseguido es identificar a cada sujeto productor si lo hubiere dentro de un mismo productor generador del fondo.

En el caso específico del Archivo Provincial, es preciso aclarar que parecería que la institución es la misma y que solo ha sufrido un proceso de evolución en el tiempo y aunque también, en la práctica no es del todo así. Algunas funciones parecen mantenerse pero no se comportan exactamente iguales. También se alteran el poder de decisión de los Superiores y el alcance de las mismas. Estas son las razones fundamentales que determinaron el hecho de constituir un fondo para cada momento histórico por el que transcurrió la institución. Paralelamente al proceso de identificación se llevó a cabo la limpieza mecánica de la documentación, así como la eliminación de duplicados, borradores, fotocopias y minutas.

Posteriormente, en la continuación de la fase de identificación, dentro de los fondos se constituyeron los subfondos inherentes si los hubo y se delimitaron preliminarmente las series, así como algunas unidades documentales simples o compuestas no seriadas. Los subfondos casi siempre responden a los diferentes sujetos productores dentro del mismo productor o a agrupaciones de personas que funcionaban dentro de una misma instancia. Por ejemplo, dentro del Fondo Comunidad de Tolosa SSS en el Archivo homónimo, existen los subfondos Seminario Menor, Noviciado, Postulantado y otros.

Por su parte, las unidades documentales no seriadas definidas como tal, aunque dentro de un fondo o subfondo, en algunos casos no siempre constituyen series. Ello obedecía al hecho de ser tipologías documentales empleadas una sola vez en trámites excepcionales, que no perduraron en el tiempo y que fueron fruto de una gestión transitoria.

Una vez conocidas exhaustivamente las funciones de cada Comunidad sacramentina y de la estructura de sus fondos, se pudo comprobar que en el caso de estos Archivos predominan los expedientes, los informes, las actas y las cartas como principales tipos documentales.

El conocimiento de cada uno de estos aspectos en la fase de identificación repercutió favorablemente en los procesos de valoración y de organización archivísticas que siguieron. No obstante, esta relación no fue unidireccional sino que, de conjunto con la valoración y la organización archivísticas (a través de la clasificación y ordenación), fue necesario revisar lo realizado en la fase de identificación.

A la par que se realizaba el proceso de identificación, se inició el de valoración. La determinación de las categorías, del flujo de información y de los documentos y de la estructura más general de los fondos en cada Archivo, labores propias de la identificación, contribuyeron también a definir los valores primarios y secundarios de los documentos.

El mismo procedimiento se aplicó también con mayor profundidad junto a la fase de organización. En estos momentos del proyecto la valoración no destacó por 
su contribución a la selección para la posterior eliminación y expurgo documental sino por el minucioso y cuidadoso estudio de los valores de cada documento. El objetivo radicaba en no despreciar algo que tuviera un mínimo de valor o interés. Aunque se retomará más adelante, como resultado de esta fase se recomendó la conservación permanente de aproximadamente el 90\% del volumen documental de todo el Sistema de Archivos por su valor secundario.

El 10\% depurado, correspondió a segundas copias, copias de borradores, transcripciones sin firmar ni sellar, fotocopias múltiples y otros documentos que engrosaban inútilmente los expedientes y series. Llegados a este punto se produce un proceso curioso porque la valoración documental no consiste en la depuración y expurgo de los expedientes y series pero son acciones que se hacen durante esta fase.

No obstante, al ser Archivos privados y carecer de Comisiones de Valoración, más allá de la que puedan formar el propio archivero y los superiores religiosos, lo definido con valor primario y secundario, así como lo determinado como expurgo, siempre constituyeron propuestas a aprobar, lo que indujo a recopilar por separado, todo lo suceptible a expurgar desde el punto de vista archvístico, a la vez que se le asignaba el valor a cada unidad documental, expediente o serie. Ello es una caracteística típica de la valoración en este tipo de archivos: que puede llegar a ser a nivel de documento.

Conviene aclarar que la fase de valoración, era la primera vez que se realizaba en todos los fondos de estos Archivos. No existía un criterio uniforme sobre qué eliminar ni cuándo hacerlo. No existía un plan de conservación ni de eliminación documental aunque sí hubo preocupación y ocupación en conservar todo lo que se consideraba con un mínimo de valor.

Al realizarse el proceso de reorganización archivística que se describe en este trabajo, se realizó la fase de valoración a todo el volumen documental del Sistema de Archivos de una sola vez, depurándose las series documentales que habían sido transferidas a los Archivos. Estos funcionan como Archivos Históricos porque no conservan documentos que están en fase de tramitación o que tienen aún valor probatorio (alrededor de 5 años). Ello fue lo que permitió realizar la valoración a todo el volumen documental en su conjunto y expurgar un $10 \%$ de ese volumen.

El resto de series valoradas, deben conservarse íntegramente porque contienen información valiosa de tipo procedimental, organizacional, doctrinal, patrimonial o simplemente histórico-informativa, de ahí la recomendación de su preservación.

Como ya se enunció, al recopilar todo lo propuesto para expurgo en espera de la autorización para su eliminación definitiva, se puedo apreciar que ello cnstituyó, aproximadamente un $10 \%$ del volumen documental total. Contrariamente a lo que pasa en las Administraciones públicas, la Administración privada como es el caso de la congregación religiosa que nos ocupa, "parece ser", lo que se pudiera definir como más eficiente, o al menos más austera, y eso es quizás lo que determina que el volumen documental expurgado para su eliminación, sea relativamente poco, o visto de otra manera, en la administración de esta congregación religiosa, no era ni es característico una producción documental cuyo fin sería o será luego su eliminación.

Ello es también la causa en la cual radica el valor asignado a cada unidad documental o serie, ya que el valor de los documentos consiste en ser pruebas 
testificales de gestiones y trámites, memorias de procedimientos que fueron característicos de épocas anteriores pero incluso, evidencias de la evolución que han tendo las propias ideas y concepciones religiosas.

Ello trató de reflejarse también en los cuadros de clasificación elaborados luego de la identificación y valoración documentales. A modo de ejemplo, en la Tabla 2 se puede apreciar el cuadro de clasificación obtenido para el Archivo Provincial. Se invita a apreciar de ese cuadro, el punto 2.9 referido a las "notificaciones de misas".

Esta es una serie que perduró durante los años 50, 60 y 70 del siglo pasado. Es una serie y una tipología documental que ya no se emplea en la actulidad, sin embargo, es una serie a conservar permanentemente por ser expresión de la forma de organización de esta actividad, de justificación económica de ingresos y de la manera en que esta congregación ponía en práctica su misión de acercamiento a los fieles. Estos son algunos de los criterios que definen su valor como serie y su inclusión en el cuadro de clasificación. El mismo razonamiento es aplicable a otras muchas.

Tabla 2. Propuesta de cuadro de clasificación orgánico-funcional a nivel de serie para el Archivo Provincial de los Padres Sacramentinos.

\section{Fondo: Vice-Provincia}

1.1. Informes de visitas canónicas

1.2. Actas del Consejo Vice-Provincial

1.3. Informes de personal

1.4. Correspondencia general

\section{Fondo: Provincia de San Pedro Apóstol}

\section{a) Gobierno}

2.1. Decretos de gobierno

2.2. Circulares

2.3. Expedientes de participación en Capítulos y Consejos Generales

2.4. Expedientes de autorizaciones

2.5. Informes de visitas canónicas

2.6. Expedientes de preparación para los Capítulos Provinciales

2.7. Actas de los Capítulos

2.8. Ordenanzas de Capítulos

2.9. Notificaciones de asignaciones de misas

2.10. Expedientes de fundación de comunidades

2.11. Expedientes de asignaciones de parroquias

2.12. Expedientes de cierre de instalaciones o comunidades

2.13. Expedientes de supresión de comunidades

2.14. Reglamentos

2.15. Informes de la Provincia

2.16. Memorias de la Provincia

2.17. Crónicas de la Provincia

2.18. Actas del Consejo Provincial

2.19. Correspondencia general

b) Personal

2.20. Informes del personal 
2.21. Registros de religiosos y escolásticos

2.22. Informes de aniversarios de profesión u ordenación

2.23. Expedientes personales de religiosos

2.24. Listados de religiosos difuntos

c) Actividades

2.25. Actas de Reuniones

2.26. Expedientes de participación en actividades

2.27. Programas de actividades

2.28. Memorias de actividades

d) Economía

2.29. Reportes económicos

2.30. Informes económicos

e) Patrimonio

2.31. Inventarios de bienes

2.32. Expedientes de compra y venta de bienes

2.33. Expedientes de convenios

2.34. Expedientes de acuerdos

f) Impuestos y tributaciones

2.35. Resguardos de la declaración y pago de impuestos

2.36. Informes de valoración de bienes

g) Gastos

2.37. Comprobantes de pago

2.38. Registro de gastos

h) Subfondo Comunidad de Barcelona SSS

- Informes anuales

- Informes económicos

- Registros de gastos

- Actas de reuniones

- Contratos de suministros y arrendamiento

- Correspondencia general

i) Subfondo Comunidad de Bilbao-Deusto SSS

j) Subfondo Comunidad de Bilbao-Zurbaran SSS

k) Subfondo Comunidad de Bogotá SSS

l) Subfondo Comunidad de Caguas SSS

m) Subfondo Comunidad de Cali SSS

n) Subfondo Comunidad de Écija SSS

o) Subfondo Comunidad de La Habana SSS

p) Subfondo Comunidad de Madrid-Sáinz de Baranda SSS

q) Subfondo Comunidad de Madrid-Santa Hortensia SSS

r) Subfondo Comunidad de Madrid-Corazón de María SSS

s) Subfondo Comunidad de Medellín SSS

t) Subfondo Comunidad de Pamplona SSS

u) Subfondo Comunidad de Popayán SSS

v) Subfondo Comunidad de Tolosa SSS

w) Subfondo Comunidad de Villaro SSS

${ }^{2}$ Las series son similares en cada Comunidad, de ahí que no se repitan. 


\section{x) Subfondo Región Juan XXIII SSS}

\section{Fondo Delegación de la Provincia de Nuestra Señora del Santísimo Sacramento}

3.1. Expedientes de nombramientos

3.2. Expedientes de convocatoria para la elección del Delegado

3.3. Expedientes de participación de la Delegación en los Capítulos Provinciales

3.4. Expedientes de Asambleas de la Delegación

3.5. Correspondencia general

Fuente: Toledo Díaz, Edison. Reelaboración, julio de 2015.

El cuadro de clasificación constituyó además una guía necesaria que condujo a la propia jerarquización de los niveles de macrodescripción (fondos, subfondos y series) y a la estructuración intelectual de los documentos en cada Archivo. Además, sentó bases muy importantes para la descripción multinivel posterior.

Aunque sólo se ha mostrado uno de los cuadros de clasificación obtenidos, conviene resaltar también que todos los elaborados fueron orgánico-funcionales. En ellos se retomaron las diferentes divisiones o secciones orgánicas de cada Comunidad. Luego se agruparon las series en torno a esas secciones teniendo en cuenta las funciones, actividades, trámites y gestiones que se llevan a cabo .

En la bibliografía consultada se valoraron algunos cuadros de clasificación fundamentalmente de Archivos eclesiásticos parroquiales, diocesanos y catedralicios. No obstante, se reconoce que la normalización de la clasificación implicará mucho más tiempo de estudio y análisis, máxime si se tiene en cuenta que siendo todos de la Iglesia Católica, son instituciones con fines muy delimitados y específicos. Lo mismo sucede incluso hacia el interior de una misma institución como la que nos ocupa, con estructuras muy amplias y extendidas.

Finalizada la ordenación de todas las series en cada uno de los Archivos, preliminarmente se obtuvieron como resultado 21 conjuntos documentales correspondientes al nivel de fondo y 52 subfondos, reunidos en los 5 Archivos que forman el Sistema de Archivos de los Padres Sacramentinos en España. También se obtuvieron preliminarmente más de 1100 series, 900 subseries y 1000 unidades documentales no seriadas entre los cinco Archivos. Se era consciente de que estas cantidades se precisarían al concluir la descripción de los fondos en cada Archivo. En la determinación de las series influyeron tres aspectos fundamentalmente: que los documentos fueran realizados por el mismo sujeto productor, en el ejercicio de una misma función y que constituyeran iguales tipologías documentales.

En cuanto a las series como tal, desde la perspectiva de esta institución que dirige un proceso en cuestión y que emplea recursos de todo tipo, su producción documental no varía en demasía a las de cualquier otra institución. De ahí que destaquen series como los expedientes de personal, los expedientes de gestión con el patrimonio, los expedientes de reuniones, los informes de personal, los informes económicos y las memorias anuales.

Sin embargo, es preciso destacar también otras series que son más características de la condición de institución privada dedicada al culto, por lo 
que incluyen una producción documental que es expresión, no ya de una gestión administrativa, sino de concepciones personales, sentimientos, pensamientos y demás, y que prueban, la manera en que los religiosos entendieron y entienden su ideal. Aquí se destacan series como las crónicas, oraciones, jaculatorias, diarios y demás.

Por otro lado, las tipologías documentales que se agruparon en torno a una misma serie, en ocasiones no respondían a unas mismas características formales o de formato. Se imponía entonces estudiar detenidamente cada documento para comprobar que se trataba del mismo tipo. El cambio de estas características formales, -que no de contenido-, respondía a cambios en las personas que dirigían la institución, de evoluciones naturales de las funciones o de cambios para lograr mayor eficacia y eficiencia documental.

La agrupación de los documentos en sus respectivas series, gracias a la ordenación y regidas por el cuadro de clasificación, garantizó una mayor eficiencia de la siguiente fase del proceso archivístico que es la descripción. No obstante, ello no quiere decir que la ordenación concluyera definitivamente. Junto a la descripción archivística, hubo que retomar la ordenación, por ejemplo, cuando se ordenaron las unidades documentales ya fueran simples o compuestas dentro de las series o cuando se ordenaron las unidades simples dentro de las compuestas.

La práctica continuó demostrando que en un proceso sistémico como la gestión archivística en Archivos privados religiosos, tal y como la entiende también el autor de este trabajo, las fases se entrecruzan y alternan: o sea, se complementan. Habrá que recurrir a ellas tantas veces como se necesiten. Ello motiva que los resultados de cada fase sean relativamente variables y susceptibles de perfeccionarse y rectificarse durante toda la reorganización archivística de este tipo de Archivos.

Para la descripción de los fondos de los Archivos de los Padres Sacramentinos, se diseñó una base de datos relacional que incluyó todos los campos de la Norma Internacional General de Descripción Archivística, ISAD(G). Con la base de datos diseñada, se generó luego un formulario que también incluyó los campos que propone la Norma $\operatorname{ISAD}(\mathrm{G})$. Por medio de este formulario, se introdujeron los elementos descriptivos como tal a la base de datos. En la Figura 9 se puede apreciar un fragmento de ese formulario con algunos de los campos de la Norma y parte de una descripción realizada. 


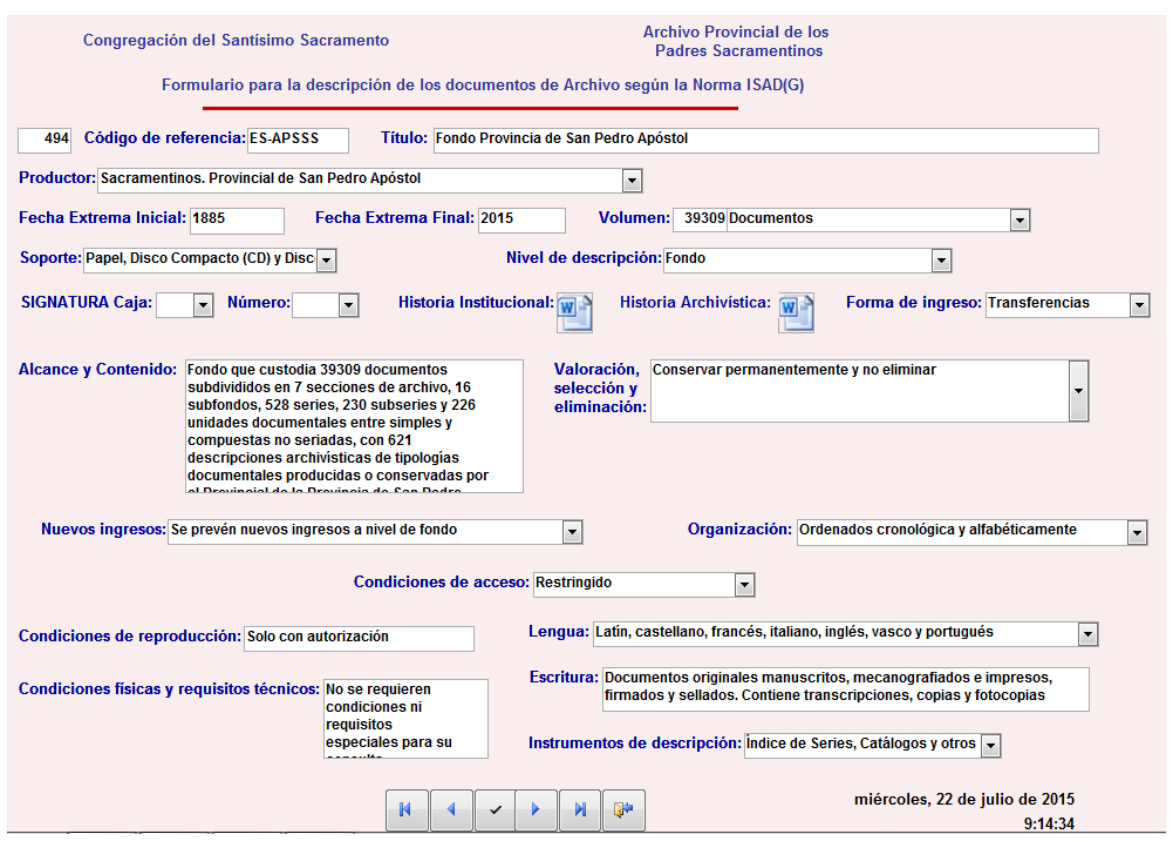

Figura 9. Sección del formulario con una descripción archivística iniciada, correspondiente al Fondo Provincia de San Pedro Apóstol.

Fuente: Toledo Díaz, Edison. Elaboración del autor. Abril de 2012.

Según el proceso de descripción, el Sistema de Archivos de los Padres Sacramentinos contiene alrededor de 130 mil documentos dispuestos para su conservación permanente y listos para su acceso y consulta por los usuarios internos. El proceso de valoración permitió definir los documentos con valor secundario y separar y expurgar alrededor de un $10 \%$ del volumen total de documentos con que contaban inicialmente los Archivos, que a su vez disponen en la actualidad de más de 2300 descripciones archivísticas normalizadas.

Los documentos expurgados consistían fundamentalmente en segundas copias, borradores y trascripciones de originales que engrosaban los expedientes innecesariamente. Por ello se propuso su eliminación definitiva previa constatación de que en los Archivos quedaban conservados el original y copias en los casos requeridos.

Junto al proceso de descripción se fue completando el proceso de ordenación. Los criterios de ordenación más empleados fueron el cronológico, el alfabético o ambos a la vez. Por ejemplo, la serie "Actas de los Capítulos Provinciales..." siguen una ordenación cronológica, mientras que la serie "Expedientes personales de los religiosos..." tienen una ordenación primero alfabética y luego cronológica dentro de cada expediente.

Una vez ordenadas las unidades documentales se colocaron en carpetas o subcarpetas en dependencia de la necesidad, se etiquetaron, se introdujeron en las 
cajas, se etiquetaron estas también y se instalaron en las estanterías. Con ello se finalizaba la organización como fase del tratamiento archivístico (Ver Figura 10).
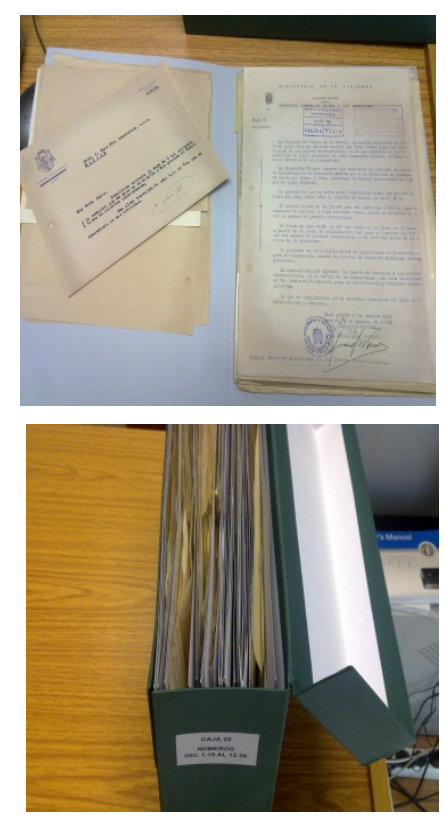
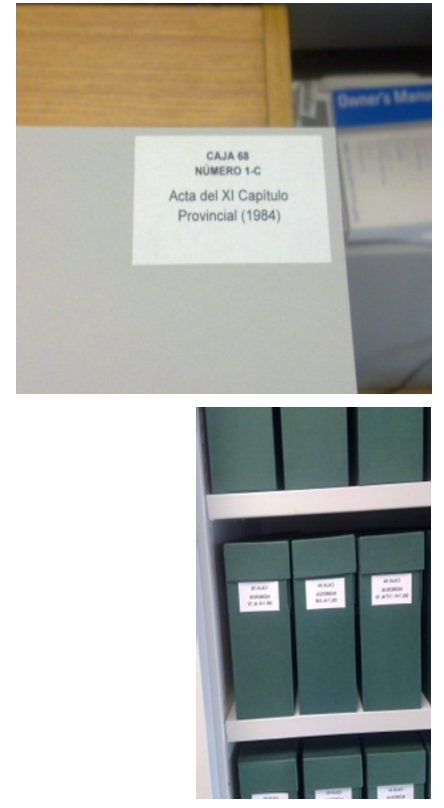

Figura 10. Fotos de algunos de los momentos del proceso de organización (ordenación) e instalación de las unidades documentales para su conservación permanente en el Archivo Provincial de los Padres Sacramentinos. Fuente: Toledo Díaz, Edison. Enero de 2012.

Los materiales empleados para la ordenación e instalación de los documentos como parte del proceso de la organización pero inherente también a la conservación, -que es transversal a todo el tratamiento archivístico-, fueron las carpetas, subcarpetas, cajas de archivo y etiquetas. De todo este material se adquirieron las cantidades necesarias.

Las carpetas son de cartulina de archivo de 240gr. Por su parte las camisas o subcarpetas son para la conservación permanente también, fabricadas con papel de archivo de 80gr. Tanto las carpetas como las subcarpetas, son apropiadas para documentos de tamaño FOLIO+ ya que sus medidas cerradas son de $365 \times 250 \mathrm{~mm}$ y abiertas son de $365 \times 500 \mathrm{~mm}$. Los materiales de fabricación de las carpetas y las subcarpetas cumplen la Norma ISO 9706 en cuanto a conservación permanente para archivos, museos y bibliotecas y han superado el Photographic Activity Test (P.A.T.). Es material libre de ácidos.

Las etiquetas son de 70x40mm, de color blanco. Su adhesivo no es perjudicial para los documentos. Estas etiquetas solo se colocaron en el exterior de las carpetas, subcarpetas y cajas. En cada etiqueta se plasma la signatura. En esta, el primer número corresponde a la CAJA y el segundo, al NÚMERO dentro de la caja. Ejemplo: la SIGNATURA 2.10 significa que el documento está en la CAJA 2 
y es el Número 10 dentro de la caja. La numeración empleada en las etiquetas (así como la ordenación misma) está en correspondencia con el cuadro de clasificación y es continua. De esa forma, resulta muy difícil que se repitan los números de las cajas o los de las carpetas en un mismo Archivo.

Todas las cajas son instaladas en estanterías que tienen una altura promedio de $2.90 \mathrm{~m}$. En cada sección de las estanterías hay entre 5 y 6 baldas y en cada balda hay alrededor de 7 cajas, lo que hace un aproximado de 35-40 cajas por sección. Cada sección está identificada en el exterior (Ver Figura 11).
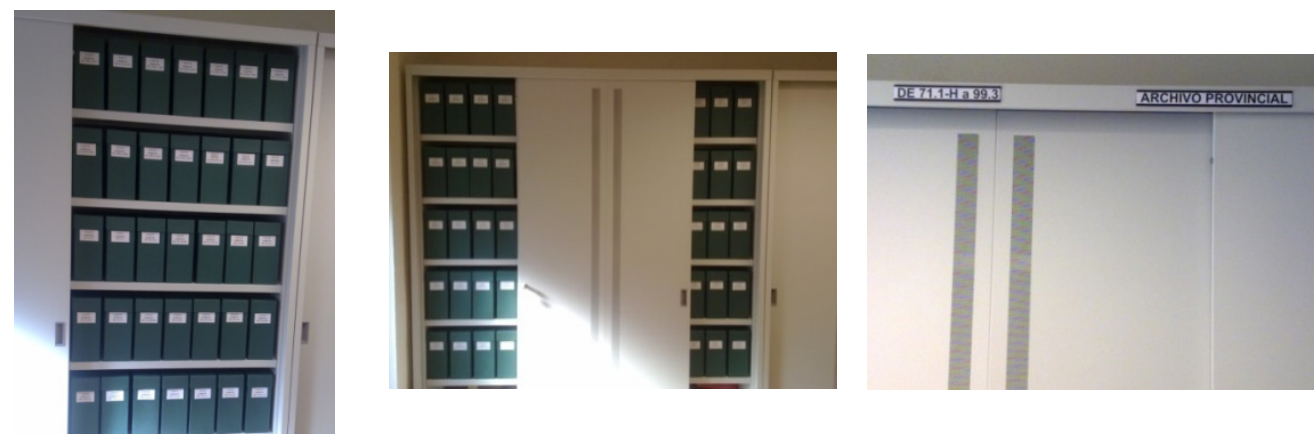

Figura 11. Fotografías que muestran secciones del Archivo Provincial totalmente instaladas para la conservación permanente de los documentos.

Fuente: Toledo Díaz, Edison. Abril 2012.

Resultan evidentes los puntos de contacto que se producen entre las fases de organización y descripción archivísticas con las acciones para la conservación de los documentos. Lo mismo sucede también entre la fase identificación y la conservación aunque no se haya especificado mucho en ello. La relación entre las fases del tratamiento archivístico y la conservación es lo que propicia que esta última sea transversal a toda la gestión archivística.

Además de las ya enunciadas, otras actividades de conservación, en este caso preventivas, llevadas a cabo durante el tratamiento archivístico fueron la impieza mecánica en seco de los documentos para retirar polvo y suciedades, el desdoblado y/o alisado de los documentos, retirada de grapas y eliminación de otros materiales, elementos plásticos y/o de metal, adhesivos y pegamentos empleados en la sujeción de los documentos.

De manera global, los principales resultados de la reorganización archivística llevada a cabo en el Sistema de Archivos de los Padres Sacramentinos en España se han resumido en la Tabla 3. 
Tabla 3. Principales resultados obtenidos en el procesamiento archivístico de los Archivos de los PP. Sacramentinos.

\begin{tabular}{|c|c|c|c|c|c|c|}
\hline & $\begin{array}{c}\text { Archivo } \\
\text { Provincial } \\
\text { San Pedro } \\
\text { Apóstol, } \\
\text { Madrid }\end{array}$ & $\begin{array}{c}\text { Archivo } \\
\text { Comunidad } \\
\text { de Tolosa } \\
\text { SSS }\end{array}$ & $\begin{array}{c}\text { Archivo } \\
\text { Comunidad } \\
\text { de } \\
\text { Pamplona } \\
\text { SSS }\end{array}$ & $\begin{array}{c}\text { Archivo } \\
\text { Comunidad } \\
\text { de Madrid- } \\
\text { Santa } \\
\text { Hortensia } \\
\text { SSS }\end{array}$ & \begin{tabular}{|c|} 
Archivo \\
Comunidad \\
de Madrid- \\
Sáinz de \\
Baranda \\
SSS
\end{tabular} & $\begin{array}{c}\text { Total del } \\
\text { Sistema } \\
\text { de } \\
\text { Archivos }\end{array}$ \\
\hline $\begin{array}{l}\text { Fecha de } \\
\text { reorganización }\end{array}$ & $\begin{array}{c}\text { 2011- } \\
2012 \text {; } \\
2015 \text { y } \\
2016\end{array}$ & 2012-2014 & 2014 & 2015 & 2016 & $\begin{array}{l}2011- \\
2016\end{array}$ \\
\hline Fondos & 3 & 12 & 2 & 2 & 2 & 21 \\
\hline Subfondos & 17 & 27 & 7 & $\mathbf{0}$ & 1 & 52 \\
\hline Series & 274 & 543 & 147 & 49 & 82 & 1177 \\
\hline Subseries & 234 & 489 & 118 & 40 & 57 & 938 \\
\hline $\begin{array}{l}\text { UDS y UDC } \\
\text { no seriadas }\end{array}$ & 238 & 561 & 125 & 45 & 41 & 1010 \\
\hline $\begin{array}{l}\text { Total de } \\
\text { documentos }\end{array}$ & 50114 & 63566 & 7980 & 3804 & 10676 & 136140 \\
\hline Fecha inicial & 1885 & 1881 & 1927 & 1964 & 1915 & 1881 \\
\hline Fecha final & 2016 & 2014 & 2014 & 2014 & 2009 & 2016 \\
\hline $\begin{array}{l}\text { Total de cajas } \\
\text { de archivo }\end{array}$ & 154 & 372 & 68 & 34 & 32 & 660 \\
\hline $\begin{array}{l}\text { Cantidad de } \\
\text { descripciones }\end{array}$ & 681 & 1150 & 291 & 101 & 136 & 2359 \\
\hline
\end{tabular}

Fuente: Toledo, Edison. Datos del autor, enero de 2016. 
Lo que gráficamente puede mostrarse tal como sigue en la Figura 12:
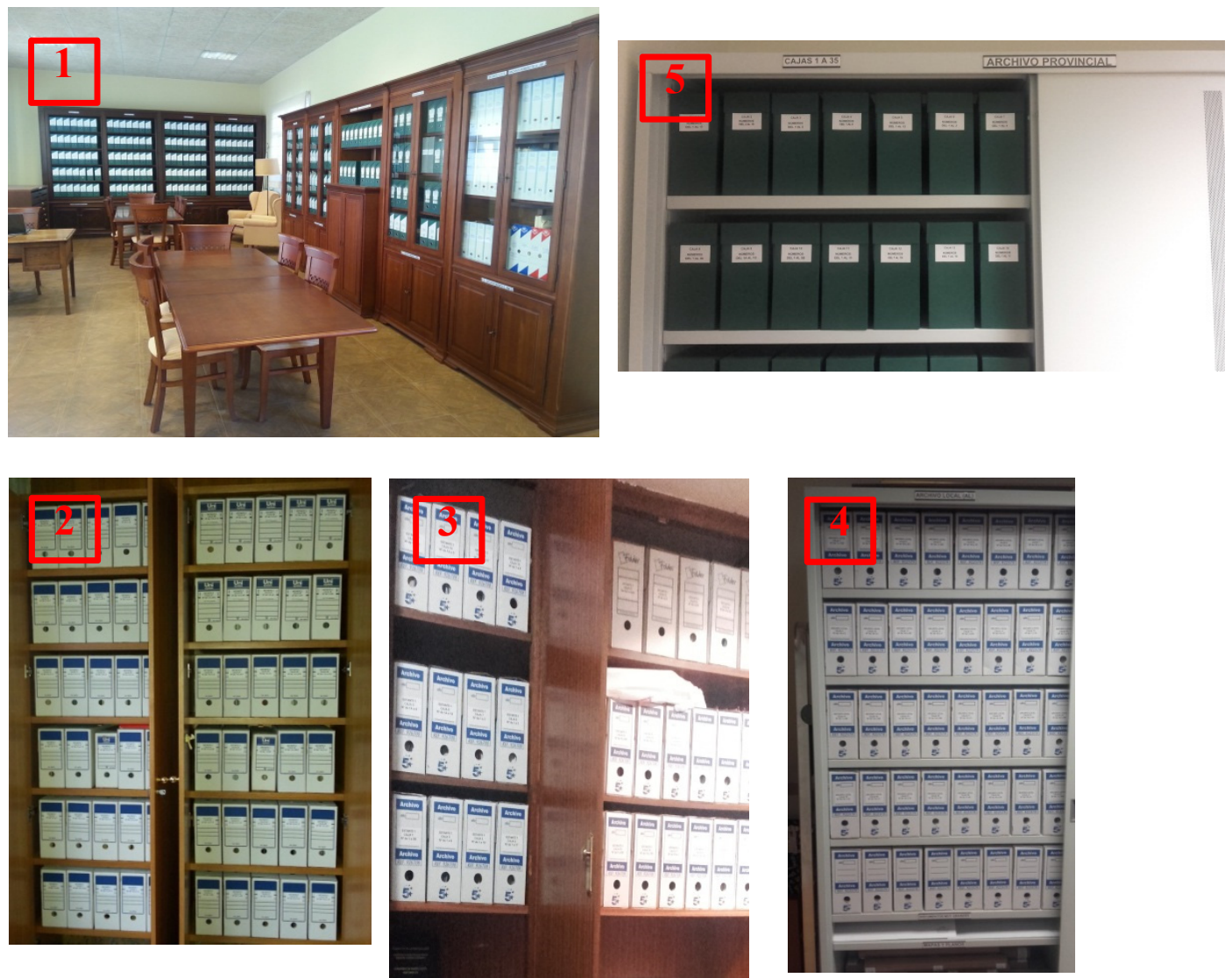

Figura 12: Archivos organizados del Sistema de Archivos de los Padres Sacramentinos en España: 1- Archivo Local de la Comunidad de Tolosa SSS. 2- Archivo Local de la Comunidad de Pamplona SSS. 3- Archivo Local de la Comunidad de Madrid-Santa Hortensia SSS. 4- Archivo Local de la Comunidad de Madrid-Sáinz de Baranda SSS. 5Archivo Provincial de los Padres Sacramentinos en Madrid. Fuente: Toledo, Edison. Fotografías del autor, enero 2016

\section{Conclusiones}

La Vice-Provincia de España (1946-1949), luego Provincia de San Pedro Apóstol (1949-2011) y desde enero del año 2012 la Delegación de España de la Provincia de Nuestra Señora del Santísimo Sacramento de Italia, cuenta con un Sistema de Archivos donde se agrupan los más de 130 mil documentos producidos y recibidos por todo el sistema organizacional a lo largo de su devenir, evolución y transformación. Ello permite inferir que: 
1. El volumen documental es de alrededor de $77 \mathrm{~m}$ lineales, el cual está ordenado archivísticamente y listo para su consulta, lo cual es posible gracias a sus más de 2300 descripciones multinivel y normalizadas por medio de la Norma ISAD(G) del Consejo Internacional de Archivos (ICA).

La organización archivística de los fondos de este Sistema de Archivos se realizó según las cuatro fases del tratamiento archivístico, a saber: identificación, valoración, organización (clasificación y ordenación que le son inherentes) y la descripción. Reconocer estas fases conlleva:

2. Concebirlas como subprocesos que en su conjunto componen lo interpretado como una gestión archivística. Ello posibilita también la complementariedad de cada fase y su interrelación sistémica.

Además, se precisa delimitar también que las definidas como fases, procedimientos, pasos, procesamiento e incluso gestión archivística, -que no del Archivo-, resultará necesario hacerlo en relación con el marco teórico y con los fundamentos metodológicos de la Archivística. Ello propicia:

3. La obtención de múltiples ventajas para la organización de cualquier Archivo, aún privado y religioso. Esto no limita ni impide que haya acciones que especifiquen, particularicen y contextualicen la práctica según el tipo de Archivo, tal y como resultó en este caso.

De manera que las fases o subprocesos archivísticos de esta gestión son perfectamente aplicables a un archivo privado, religioso y de la Iglesia Católica, Más allá del nombre que se le dé a las acciones y tareas inherentes a la organización de los archivos, su objetivo final o fin fundamental debe consistir en la accesibilidad de los documentos. Ello permite:

4. Poner el documento en función del usuario, o sea, accesible para su consulta. Esa es la nueva propiedad o cualidad resultante de una concepción procesal y sistémica de lo que se considera una gestión archivística. A su vez, ésta debiera ser medible a través del acceso al documento, incluido en el tipo de Archivos abordado en este trabajo.

Aunque todos los empeños resultan válidos, la satisfacción de cualquier institución no debiera estar en realizar alguno de los procesos de la gestión archivística por separado o aislado. No se llegaría a la nueva cualidad del sistema de la gestión archivística que, se insiste, consiste en el acceso y consulta de los documentos conservados.

Por ello, el mejor de los resultados de este trabajo es que:

5. El Sistema de Archivos de los Padres Sacramentinos es ya accesible. Ello a su vez ha sido también la mejor de las respuestas a la problemática expuesta por el profesor Germán García Ruipérez, y enunciada al inicio de este trabajo, la cual guió la reorganización archivística del Sistema de Archivos de los Padres Sacramentinos en España y la posterior redacción de estas líneas. 


\section{Referencias bibliográficas}

Arzobispado de Zaragoza (1995). Proyecto de organización de archivos eclesiásticos aragoneses. En: Monografias 9. Zaragoza: Aragonia Sacra. Cajalon

Congregación del Santísimo Sacramento (2009). Regla de Vida. Madrid.

Consejo Internacional de Archivos (2000). Norma Internacional General de descripción archivística. ISAD(G). Madrid: Ministerio de Educación, Cultura y Deporte, Secretaría de Estado de Cultura.

Cruz Mundet JR (2012). Manual de Archivística. Madrid: Fundación Germán Sánchez Ruipérez.

De Diego N. (2000) Los archivos españoles de la Iglesia Católica. Madrid: Cuadernos de Historia Moderna, 25, p:339-372.

De La Cruz Herranz LM, Cruces Blanco E Y Cayetano Martín MC. Archivos y sistemas. En: Escuela Iberoamericana de Archivos. Experiencias y Materiales. España: Ministerio de Educación, Cultura y Deporte.

Farías I (2005). Archivos Eclesiásticos y Religiosos: Límites y desafíos ante la Sociedad de la Información y el Conocimiento. Argentina: 2do. Congreso Argentino de Archivística. Tecnología de la Información en los Archivos, [Ponencia] $<\mathrm{http}: / / \mathrm{www}$. mundoarchivistico.com/?menu=articulos\&id=84>

García Ruipérez M (2007) Tipologías. Series documentales. Cuadros de clasificación. Cuestiones metodológicas y prácticas. Las Palmas de Gran Canaria: Asarca Forma 2. Anroart ediciones.

Heredia A (2007) ¿Qué es un archivo? Gijón: TREA.

Kaufmann, JL (2001). Pastoralidad del Archivo Eclesiástico: elementos básicos. La Plata (Buenos Aires): Universidad Católica de la Plata.

La Torre Merino JL, Martín-Palomino Y Benito M (2000). Metodología para la identificación y valoración de fondos documentales. En: Escuela Iberoamericana de Archivos. Experiencias y Materiales. España: Ministerio de Educación, Cultura y Deporte.

Lanfrey, A (2003). Les Archives Diocésaines et Congréganistes entre Conservation, Commémoration et Histoire. En: Paris: A.A.E.F. - N. 60.

Mateos, MC (2002). Un cuadro de clasificación para los archivos de la Congregación Cisterciense de San Bernardo. En: Tabula: revista de Archivos de Castilla y León. Salamanca N. 5.

Ministerio de Cultura (1995) Manual de Terminología Archivística. $<$ http://www.mcu.es/archivos/docs/MC/DTA/libro_terminologia.pdf $>$

Pedrals i Costa, X (1991). Una proposta de classificació dels Arxius Eclesiàstics Locals, segons un esquema orgànic $\mathrm{i}$ funcional: estudi de l'Arxiu eclesiàstic de Bagà. En: Lligall. Revista Catalana d'Arxivistica. Barcelona, n. 4.

Pérez MG (2006) Documentación conventual en el archivo diocesano de Mérida-Badajoz: Confección de un sistema de información histórica (localización, análisis documental y gestión automatizada). Universidad de Extremadura. Facultad de Biblioteconomía y Documentación.

Pérez MG, Vivas A (2008) Ensayo de organización de la documentación conventual: propuesta de cuadro de clasificación. En: Anales de Documentación. (11).

Ricousse, F (2000). Les Archives Des Frères Des Ecoles Chrétiennes En France archives Lasalliennes. En: Paris : A.A.E.F. - N. 54.

Taboada F y Nielsen M (2006). Archivística y normalización. Norma ISO 15489. Buenos Aires, Alfagrama Ediciones.

Toledo Díaz E (2009). Modelo de gestión del conocimiento para los trabajadores del Archivo Nacional de la República de Cuba. La Habana, [Tesis de Doctorado] 
Toledo Díaz E (2012) El Archivo de la Provincia de San Pedro Apóstol de los Padres Sacramentinos. Resultados y experiencias del procesamiento archivístico del fondo documental de una orden religiosa del clero regular. $<$ http://dspace.unav.es/dspace/bitstream/10171/23123/1/\%28Edison\%20Toledo\%29\%2 0Archivo\%20Univ\%20Navarra\%20Final\%20Julio\%202012.pdf >

Toledo Díaz E (2016). Algunas características de la identificación y clasificación archivística en la organización de fondos documentales religiosos católicos. El caso de los Archivos de los Padres Sacramentinos en España. <http://www.documentalistas.org/rd39/>

Vega Blanco, M. Síntesis del Cuadro de Clasificación de Documentos Archivo Eclesiástico-Curia Metropolitana. En: el Archivo Boletín del Archivo Eclesiástico de la Curia Metropolitana. San José (Costa Rica), n. 13. 